\title{
Heparanase Is Essential for the Development of Acute Experimental Glomerulonephritis
}

\author{
Marjolein Garsen, ${ }^{*}$ Marilen Benner, ${ }^{*}$ Henry B. Dijkman, ${ }^{\dagger}$ Toin H. van Kuppevelt, ${ }^{\ddagger}$ Jin-Ping Li, ${ }^{\S}$ Ton J. Rabelink, ${ }^{\natural}$ \\ Israel Vlodavsky, Jo H.M. Berden, ${ }^{*}$ Angelique L.W.M.M. Rops, ${ }^{*}$ Michael Elkin, ** and Johan van der Vlag *
}

\begin{abstract}
From the Departments of Nephrology, * Pathology, ${ }^{\dagger}$ and Biochemistry, ${ }^{\ddagger}$ Radboud University Medical Center, Nijmegen, the Netherlands; the Department of Medical Biochemistry and Microbiology, ${ }^{\S}$ Uppsala University, Uppsala, Sweden; the Department of Nephrology, ${ }^{\boldsymbol{\top}}$ Leiden University Medical Center, Leiden, the Netherlands; the Cancer and Vascular Biology Research Center, Bruce Rappaport Faculty of Medicine, Technion, Haifa, Israel; and the Sharett Institute, ** Hadassah-Hebrew University Medical Center, Jerusalem, Israel
\end{abstract}

\author{
Accepted for publication \\ December 8, 2015. \\ Address correspondence to \\ Johan van der Vlag, Ph.D., \\ Department of Nephrology \\ (480), Radboud Institute for \\ Molecular Life Sciences, \\ Radboud University Medical \\ Center, Geert Grooteplein 10, \\ 6525 GA Nijmegen, the \\ Netherlands. E-mail: johan. \\ vandervlag@radboudumc.nl.
}

\begin{abstract}
Heparanase, a heparan sulfate (HS) - specific endoglucuronidase, mediates the onset of proteinuria and renal damage during experimental diabetic nephropathy. Glomerular heparanase expression is increased in most proteinuric diseases. Herein, we evaluated the role of heparanase in two models of experimental glomerulonephritis, being anti-glomerular basement membrane and lipopolysaccharide-induced glomerulonephritis, in wild-type and heparanase-deficient mice. Induction of experimental glomerulonephritis led to an increased heparanase expression in wild-type mice, which was associated with a decreased glomerular expression of a highly sulfated HS domain, and albuminuria. Albuminuria was reduced in the heparanase-deficient mice in both models of experimental glomerulonephritis, which was accompanied by a better renal function and less renal damage. Notably, glomerular HS expression was preserved in the heparanase-deficient mice. Glomerular leukocyte and macrophage influx was reduced in the heparanase-deficient mice, which was accompanied by a reduced expression of both types 1 and 2 helper T-cell cytokines. In vitro, tumor necrosis factor- $\alpha$ and lipopolysaccharide directly induced heparanase expression and increased transendothelial albumin passage. Our study shows that heparanase contributes to proteinuria and renal damage in experimental glomerulonephritis by decreasing glomerular HS expression, enhancing renal leukocyte and macrophage influx, and affecting the local cytokine milieu. (Am J Pathol 2016, 186: 805-815; http://dx.doi.org/10.1016/j.ajpath.2015.12.008)
\end{abstract}

Proteinuria is a hallmark of many glomerular diseases and an independent risk factor for the progression of renal failure. ${ }^{1}$ Heparan sulfate (HS) is a highly negatively charged glycosaminoglycan that is covalently attached to a core protein, socalled HS proteoglycans. In seminal articles, researchers demonstrated the presence of HS in the glomerular filtration barrier (GFB), which is composed of glomerular endothelial cells covered by a glycocalyx, the glomerular basement membrane (GBM), and podocytes. ${ }^{2-4}$ Because of its negative charge, HS seems to play an important role in the chargeselective permeability of the GFB. ${ }^{5}$ Removal of HS with bacterial heparinase leads to a dramatic increase in glomerular permeability for neutral and cationic macromolecules. ${ }^{6}$ Genetic targeting of HS in the GFB compromised permselectivity and barrier function to a lesser extent, ${ }^{7-9}$ although the development of proteinuria has been associated with a reduced expression of $\mathrm{HS}$ in the GFB. ${ }^{3,5,10}$ The HS chain is composed of up to $150 \alpha(1-4)$-glucuronate- $\beta(1,4)-N$-acetylglucosamine disaccharide units that can be modified extensively. HS is characterized by an enormous structural diversity, which dictates the binding of several soluble ligands, such as cytokines, chemokines, and growth factors. $^{11,12}$ In addition, specific endothelial HS domains mediate the trafficking of leukocytes. ${ }^{13}$

In many human and experimental glomerular diseases, the reduced expression of HS in the GFB is associated with an increased expression of heparanase. ${ }^{3,14}$ Heparanase is an endo- $\beta(1,4)$-D-glucuronidase that can cleave HS side chains.

Supported by Dutch Kidney Foundation grants C09.2296, 15OI36, and KJBP 09.010 and by consortium grant CP09.03 (GLYCOREN).

Disclosures: None declared. 
Heparanase is synthesized as a preproheparanase of $68 \mathrm{kDa}$. To gain its biological activity, preproheparanase is processed in the endoplasmatic reticulum, where the signal peptide is removed, and further processed in lysosomes, where cathepsin L cleaves off a linker domain to form the active form of heparanase. ${ }^{14,15}$ Outside the kidney, heparanase is involved in cancer progression, in particular metastasis and neovascularization. ${ }^{16-18}$ Heparanase is also involved in the pathogenesis of several inflammatory disorders, such as inflammatory lung injury, rheumatoid arthritis, and chronic colitis. ${ }^{19-22}$ Recently, we demonstrated that heparanase is essential for the development of proteinuria in experimental diabetic nephropathy. ${ }^{23}$ In streptozotocin-induced diabetes, heparanase-deficient mice failed to develop proteinuria and renal damage, in contrast to their wild-type (WT) littermates. In addition, proteinuria was reduced and renal function improved by treatment with the heparanase inhibitor SST0001. ${ }^{23}$ In a follow-up study, we showed that heparanase contributes to the inflammatory cascade during the pathogenesis of diabetic nephropathy. ${ }^{24}$ Although heparanase plays a crucial role in the development of diabetic nephropathy, the exact role of heparanase in inflammatory glomerular diseases, such as glomerulonephritis, is still unknown.

Glomerulonephritis is characterized by the influx of inflammatory cells, proteinuria, hematuria, and a decline in renal function. Previous studies revealed that heparanase may be involved in the development of proteinuria in passive Heymann nephritis and in a model of accelerated antiGBM disease, because treatment of rats with a polyclonal antibody against heparanase or the heparanase inhibitor PI88 reduced proteinuria in both experimental diseases. ${ }^{25-27}$ In addition, it has been recently described that lipopolysaccharide (LPS)-induced glomerulonephritis involves an increased heparanase expression and a damaged glomerular endothelium, which is, in large part, mediated by tumor necrosis factor (TNF)- $\alpha{ }^{28}$ Interestingly, heparanase inhibition prevented glycocalyx loss and neutrophil adhesion during sepsis-induced acute lung injury, indicating that heparanase may also be involved in the influx of inflammatory cells. ${ }^{19}$ In another recent study, severe systemic sepsis was induced by cecal ligation and puncture, and it was suggested that heparanase mediated early renal dysfunction. Unfortunately, in the latter study, there was no direct proof for a reduced HS expression in the GFB mediated by heparanase, whereas the applied anti-HS antibody (3G10) suggests involvement of bacterialderived heparinases instead of mammalian heparanase. ${ }^{29}$

To elucidate the role of heparanase in the development of glomerulonephritis, we evaluated the involvement of heparanase in two experimental glomerulonephritis models, antiGBM and LPS-induced glomerulonephritis, in WT and heparanase-deficient mice. Our results indicate that heparanase drives anti-GBM and LPS-induced glomerulonephritis by enhancing the renal influx of inflammatory cells and by influencing the local cytokine production.

\section{Materials and Methods}

\section{Animals}

C57B1/6 (Harlan Laboratories, Jerusalem, Israel) and heparanase knockout mice ${ }^{30}$ in a $\mathrm{C} 57 \mathrm{Bl} / 6$ background were kept under pathogen-free conditions, housed in a temperaturecontrolled room with a 12-hour light/dark cycle, and had ad libitum access to food and water. All animal experiments were performed in accordance with, and approved by, the Hebrew University (Jerusalem, Israel) Institutional Animal Care and Use Committee.

Induction of Anti-GBM and LPS Glomerulonephritis and Determination of Albuminuria and Blood Urea Nitrogen

Experimental anti-GBM glomerulonephritis was induced as previously described. ${ }^{31}$ WT and heparanase-deficient mice, 14 to 15 weeks old, were injected in the tail vein with $7 \mathrm{mg}$ rabbit anti-mouse GBM IgG serum. Mice were sacrificed after 2 hours, 1 day, and 4 days to collect kidneys and blood. LPS glomerulonephritis was induced in 14- to 15-week-old WT and heparanase-deficient mice by an i.p. injection with $80 \mu \mathrm{g}$ LPS (O111:B4; Sigma-Aldrich Chemie, Zwijndrecht, the Netherlands). Mice were sacrificed after 2 days to collect kidneys and blood. Eight mice were used per time point. Urine was collected through a bladder punction or after 24 hours in metabolic cages. Collected kidneys were snap frozen in liquid nitrogen. Urinary albumin was measured by radial immunodiffusion (Mancini), and blood urea nitrogen and urinary creatinine concentrations were determined routinely in our clinical diagnostic facility.

\section{Immunofluorescence Staining}

Immunofluorescence staining was performed on cryosections ( $2 \mu \mathrm{m}$ thick), as described. ${ }^{31}$ Directly labeled antibodies included rat anti-mouse GR-1 (RB6.8C5)-fluorescein isothiocyanate (BD Biosciences, Alphen aan de Rijn, the Netherlands) and rat anti-mouse CD41-Alexa 488 (ITK Diagnostics, Uithoorn, the Netherlands). Unlabeled primary antibodies included CD68 (MCA1957) (Serotec, Oxford, UK) and the VSV-tagged anti-HS antibodies HS4C3, AO4B08, EW4G2, and EW3D10. ${ }^{31,32}$ Appropriate secondary antibodies include Alexa 488 antibodies (Invitrogen Life Technologies, Breda, the Netherlands) or anti-VSV-Cy3 antibody (SigmaAldrich). Capillary loops were visualized with the hamster anti-agrin antibody (MI91), ${ }^{33}$ recognized by a Cy-3-labeled antibody (Jackson ImmunoResearch Laboratories, West Grove, PA). Sections were post-fixed with $1 \%$ paraformaldehydephosphate-buffered saline and embedded in Vectashield mounting medium H-1000 (Brunschwig Chemie, Amsterdam, the Netherlands). HS was scored semiquantitatively for staining intensities on a scale between 0 and 10 ( 0 indicates no staining; 5, 50\% staining; and 10, 100\% staining) by two investigators (M.G. and A.L.W.M.M.R.). Glomerular influx of granulocytes 
and macrophages was determined by counting the number of cells per 50 glomeruli. Glomerular influx of platelets in LPS glomerulonephritis was determined by counting the number of platelets per 50 glomeruli, whereas the number of glomeruli with platelet aggregates per 50 glomeruli was counted for anti-GBM glomerulonephritis. Scoring was performed using a Leica CTR6000 microscope (Leica Microsystems, Son, the Netherlands) by two independent investigators (M.G. and M.B.) on blinded sections.

\section{Renal Histology}

Renal cryosections ( $2 \mu \mathrm{m}$ thick) were fixed in $96 \%$ ethanol and stained with periodic acid-Schiff. Sections were counterstained with hematoxylin and evaluated by an experienced nephropathologist (H.B.D.). At least 50 glomeruli per mouse were analyzed for the presence of glomerular lesions and mesangial matrix expansion. The average of glomeruli with lesions or mesangial matrix expansion was calculated.

\section{Heparanase Activity Assay}

The activity of heparanase in renal cortex was determined by a commercially available assay (AMS Biotechnology, Abingdon, UK). Briefly, $100 \mu \mathrm{L}$ tissue lysates were mixed 1:1 with reaction buffer and incubated on biotinylated HScoated plates for 1.5 hours at $37^{\circ} \mathrm{C}$. Plates were washed and incubated with Streptavidin-horseradish peroxidase for 60 minutes at room temperature. Peroxidase substrate was added, and the reaction was stopped by $0.2 \mathrm{~N} \mathrm{H}_{2} \mathrm{SO}_{4}$. Absorbance was measured at $450 \mathrm{~nm}$.

\section{Cell Culture}

Conditionally immortalized mouse glomerular endothelial cells (mGEnC-1) and mouse podocytes (MPC-5) were cultured, as previously described. ${ }^{34,35}$ Silencing of heparanase in mGEnC-1 was achieved after transfecting a heparanase shRNA construct (Qiagen, Venlo, the Netherlands) with Lipofectamine 2000 into undifferentiated mGEnC-1 and subsequent selection with G418 (Sigma-Aldrich). Where indicated, differentiated mGEnC-1 and podocytes were stimulated with $10 \mathrm{ng} / \mathrm{mL}$ TNF- $\alpha$ (Invitrogen Life Technologies) or $1 \mu \mathrm{g} / \mathrm{mL}$ LPS (Sigma-Aldrich) for 18 hours. All experiments were performed at least twice for confirmation.

\section{Transendothelial Albumin Passage}

mGEnC-1 seeded onto polyester membranes in tissue culture inserts $(0.4-\mu \mathrm{m}$ pore size; Corning Inc., Corning, NY) were, after differentiation, treated with TNF- $\alpha$ or LPS, as outlined. After 18 hours, medium in the insert was replaced by serum-free medium containing $0.5 \mathrm{mg} / \mathrm{mL}$ fluorescein isothiocyanate-labeled bovine serum albumin (SigmaAldrich), and medium in the well was replaced by serumfree medium. Aliquots were removed from the well after 1,
Table 1 Primers Used in Real-Time PCR

\begin{tabular}{|c|c|}
\hline Target & Primer sequence \\
\hline \multirow[t]{2}{*}{ HPSE } & F: 5'-GAGCGGAGCAAACTCCGAGTGTATC-3' \\
\hline & R: 5'-GATCCAGAATTTGACCGTTCAGTT-3' \\
\hline \multirow[t]{2}{*}{ TNF- $\alpha$} & F: 5'-CATCTTCTCAAAATTCGAGTGACAA-3' \\
\hline & R: 5'-TGGGAGTAGACAACGTACAACCC-3' \\
\hline \multirow[t]{2}{*}{ IFN- $\gamma$} & F: 5'-GGTGACCTTGTGACAAGCTC-3' \\
\hline & R: 5'-TGCTGTGTGGTCTGTCTGTC-3' \\
\hline \multirow[t]{2}{*}{ IL-6 } & F: 5'-TTCCTCTCTGCAAGAGACT-3' \\
\hline & R: 5'-TGTATCTCTCTGAAGGACT-3' \\
\hline \multirow[t]{2}{*}{ IL-10 } & F: 5'-GTGGAGCAGGTGAAGAGTGA-3' \\
\hline & R: 5'-TGCAGTTGATGAAGATGTCAAA-3' \\
\hline \multirow[t]{2}{*}{ IL-12 $\alpha$} & F: 5'-GGGAGAAGCAGACCCTTACAGA-3' \\
\hline & R: 5'-GGGTGCTGAAGGCGTGAA- $3^{\prime}$ \\
\hline \multirow[t]{2}{*}{ IL-12 $\beta$} & F: 5'-GGAAGCACGGCAGCAGAATC-3' \\
\hline & R: 5'-AACTTGAGGGAGAAGTAGGAATGG-3' \\
\hline \multirow[t]{2}{*}{ TNFRSF1a } & F: 5'-CCATCATTTGTAGGGATCCC-3' \\
\hline & R: 5'-TCTCAGAGCCTCGAGGATAT-3' \\
\hline \multirow[t]{2}{*}{ TNFRSF1b } & F: 5'-GAAATCCCAGGATGCAGTAG-3' \\
\hline & R: 5'-TCAGGCCACTTTGACTGCAA-3' \\
\hline \multirow[t]{2}{*}{ Sdc-1 } & F: 5'-GACTCTGACAACTTCTCTGGCTCT-3' \\
\hline & R: 5'-GCTGTGGTGACTCTGACTGTTG-3' \\
\hline
\end{tabular}

$\mathrm{F}$, forward; HPSE, heparanase; IFN- $\gamma$, interferon- $\gamma$; R, reverse; Sdc-1, syndecan-1; TNF- $\alpha$, tumor necrosis factor- $\alpha$; TNFRSF, TNF receptor.

2 , and 3 hours and replaced by serum-free medium. Fluorescence was measured with excitation at $495 \mathrm{~nm}$ and emission at $520 \mathrm{~nm}$, and the amount of albumin passing the endothelial cell monolayer was determined by a set of standard dilutions.

\section{RNA Isolation and Real-Time PCR}

Total RNA was isolated from mGEnC-1, MPC-5 podocytes, and kidney cortex using the RNeasy mini kit (Qiagen). RNA $(1 \mu \mathrm{g})$ was reverse transcribed into cDNA using the RevertAid First Strand cDNA Synthesis Kit (Thermo Scientific, Waltham, MA). One-tenth of cDNA was used as template in a real-time PCR using SYBR Green SuperMix (Roche Diagnostics, Mannheim, Germany) with gene-specific primers (Isogen Life Science, De Meern, the Netherlands) (Table 1) on the CFX real-time PCR system (Bio-Rad Laboratories, Hercules, CA). Gene expression levels were quantified using the $\Delta \Delta \mathrm{C}_{\mathrm{T}}$ method, with glyceraldehyde-3-phosphate dehydrogenase $(G A P D H)$ as the housekeeping gene.

\section{Statistical Analysis}

Values are expressed as means \pm SEM. Significance was evaluated by a one-way analysis of variance and post hoc analysis with Tukey's multiple-comparison test. Comparison of expression between two different groups was evaluated using the $t$-test. The nonparametric Spearman's rank test was used to calculate the correlation between HS expression and proteinuria. A two-way repeated-measures analysis of variance with Bonferroni post test was used to evaluate 

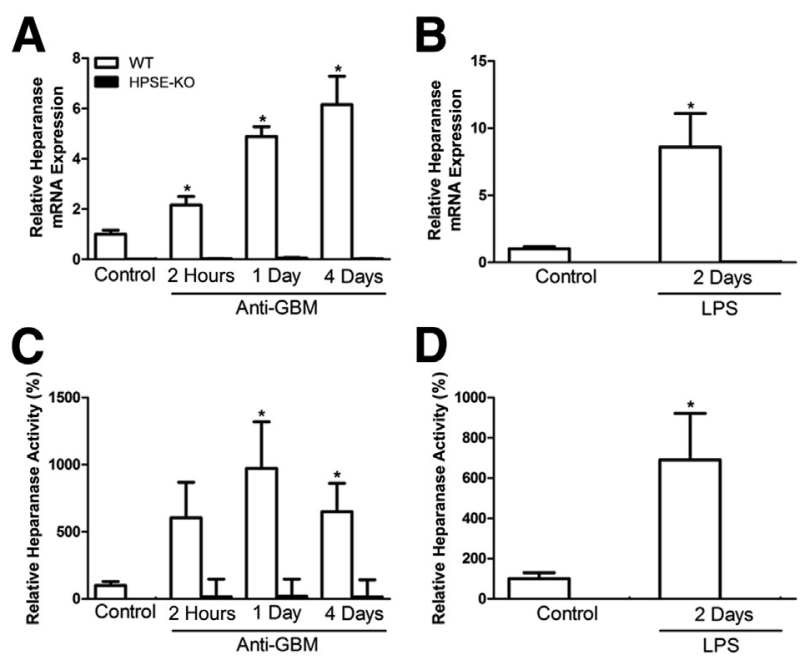

Figure 1 Experimental glomerulonephritis increases renal heparanase expression and activity in wild-type mice. Heparanase mRNA expression ( $\mathbf{A}$ and $\mathbf{B}$ ) and heparanase activity ( $\mathbf{C}$ and $\mathbf{D})$ are increased by induction of anti-glomerular basement membrane (GBM) glomerulonephritis ( $\mathbf{A}$ and $\mathbf{C}$ ) and lipopolysaccharide (LPS) glomerulonephritis ( $\mathbf{B}$ and D). Heparanase was not expressed or active in the heparanase-deficient mice. ${ }^{*} P<0.05$ versus control.

significance for the transendothelial albumin passage experiments. Statistical analysis was performed using GraphPad Prism version 5.03 (GraphPad Software, Inc., San Diego, CA). $P \leq 0.05$ was considered statistically significant.

\section{Results}

Heparanase Expression and Activity Increase by Induction of Experimental Glomerulonephritis

Heparanase mRNA expression in kidney cortex significantly increased 2 hours, 1 day, and 4 days after the induction of antiGBM glomerulonephritis (Figure 1A) and 2 days after the induction of LPS glomerulonephritis (Figure 1B). In WT mice, the increased heparanase expression correlated with an increased heparanase activity after induction of both anti-GBM and LPS glomerulonephritis, although heparanase activity did not significantly increase 2 hours after the induction of antiGBM glomerulonephritis $(P=0.09)$ (Figure $1, \mathrm{C}$ and D). Notably, we choose to evaluate day 2 for the LPS-induced model for glomerulonephritis, because we did not observe differences in the urinary albumin/creatinine ratio, nor in renal heparanase activity 1 day after administration of LPS in WT mice (Supplemental Figure S1).

\section{Heparanase-Deficient Mice Show Better Renal Function} and Less Renal Damage after Induction of Experimental Glomerulonephritis

A significant proteinuria was observed 1 and 4 days after the induction of anti-GBM glomerulonephritis (Figure 2A) and 2 days after the induction of LPS glomerulonephritis in WT mice
(Figure 2B). Proteinuria was reduced in the heparanasedeficient mice in both models, although this was borderline significant 4 days after induction of anti-GBM glomerulonephritis $(P=0.06)$. Renal function, as measured by the blood urea nitrogen concentration, was impaired 4 days after induction of anti-GBM glomerulonephritis in the WT mice, whereas it was normal in the heparanase-deficient mice (Figure 2C). By induction of LPS glomerulonephritis, renal function was significantly decreased in both WT and heparanase-deficient mice (Figure 2D). However, renal function was significantly better in the heparanase-deficient mice compared with WT mice. WT mice showed significantly more glomerular damage compared with the heparanase-deficient mice, for both the antiGBM and LPS models (Figure 3, A-C). By induction of antiGBM glomerulonephritis, WT mice showed significantly more glomerular lesions compared with the heparanase-deficient mice (Figure 3, A and B). Moreover, in the LPS model, mesangial matrix expansion, although limited, was only observed in the WT mice and not in the heparanase-deficient mice (Figure 3, A and C).

\section{Heparanase Deficiency Preserves Glomerular HS Expression in Experimental Glomerulonephritis}

Because heparanase plays an important role in HS turnover, we determined the glomerular expression of a highly
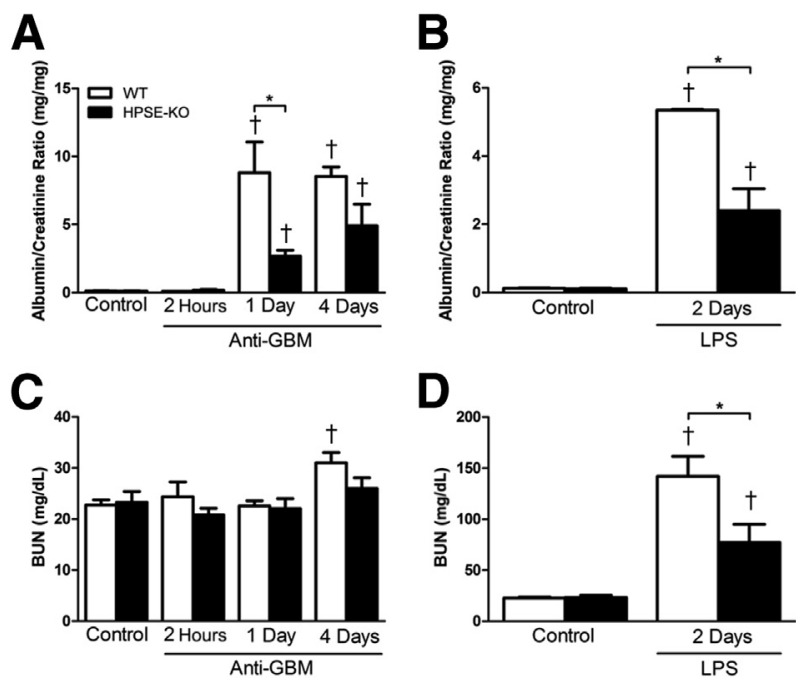

Figure 2 Heparanase-deficient (HPSE-KO) mice show better renal function during anti-glomerular basement membrane (GBM) and lipopolysaccharide (LPS)-induced glomerulonephritis. The albumin/creatinine ratio significantly increases in wild-type (WT) mice compared with untreated control mice 1 and 4 days after induction of anti-GBM glomerulonephritis (A) and 2 days after induction of LPS glomerulonephritis (B). HPSE-KO mice show a lower albuminuria at these time points $(P=0.06$ versus WT mice 4 days after induction of anti-GBM glomerulonephritis). C: Plasma blood urea nitrogen (BUN) levels significantly increase in WT mice compared with untreated control mice 4 days after induction of anti-GBM glomerulonephritis. Plasma BUN levels are normal in the HPSE-KO mice. D: Plasma BUN levels are significantly lower in the HPSEKO mice compared with WT mice 2 days after induction of LPS glomerulonephritis. ${ }^{\star} P<0.05$ versus anti-GBM/LPS-injected WT mice; ${ }^{\dagger} P<0.05$ versus control. 


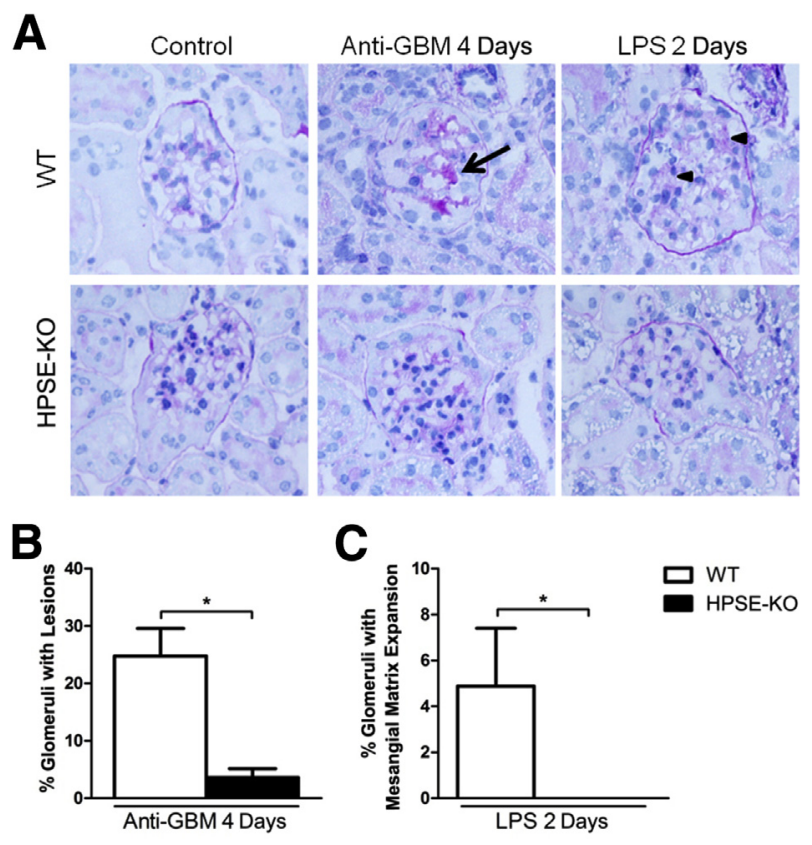

Figure 3 Heparanase-deficient (HPSE-KO) mice show less glomerular injury during anti-glomerular basement membrane (GBM) and lipopolysaccharide (LPS)-induced glomerulonephritis. A: Representative images of renal histology and semiquantitative analysis of the percentage of glomeruli with lesions (arrow; B) and glomeruli with mesangial matrix expansion (arrowheads; C), as determined by a periodic acid-Schiff stain. Wild-type (WT) mice show significantly more glomerular injury compared with HPSEKO mice 2 days after induction of LPS glomerulonephritis and 4 days after induction of anti-GBM glomerulonephritis. ${ }^{*} P<0.05$ versus anti-GBM/LPSinjected WT mice. Original magnification, $\times 400(A)$.

sulfated HS domain recognized by the anti-HS antibody HS4C3. Glomerular HS expression in WT mice was reduced 1 and 4 days after the induction of anti-GBM glomerulonephritis (Figure 4, A and B) and 2 days after the induction of LPS glomerulonephritis (Figure 4, A and C). Heparanase deficiency preserved HS expression in both models. HS expression inversely correlated with the level of proteinuria $(r=-0.79, P<0.0001)$ (Figure $4 \mathrm{D})$, which is consistent with previous results, ${ }^{10}$ but also in line with the data on heparanase expression and activity (Figure 1).

\section{Heparanase Deficiency Leads to a Reduced Glomerular} Influx of Leukocytes and Macrophages in Experimental Glomerulonephritis

Next, we evaluated the role of heparanase on renal inflammation by determining the glomerular influx of polymorphonuclear granulocytes (PMNs) and macrophages. Glomerular PMN influx is an important mediator of glomerular damage and proteinuria in the early heterologous phase of anti-GBM glomerulonephritis. Glomerular PMN influx was maximal 2 hours after the induction of antiGBM glomerulonephritis in both WT and heparanasedeficient mice and decreased over time (Figure 5A). Notably, after 2 hours, glomerular PMN influx was significantly reduced in the heparanase-deficient mice compared with the WT mice. Consistent with this, the expression of several inflammatory HS domains on endothelium that were previously shown to be important for leukocyte trafficking was also reduced in the heparanasedeficient mice (Supplemental Figure S2). ${ }^{13,31,36}$ Mice with LPS-induced glomerulonephritis did not show significant differences in glomerular PMN influx when comparing WT mice with heparanase-deficient mice (Figure 5B). Glomerular macrophage influx was increased after induction of both anti-GBM and LPS glomerulonephritis (Figure 5, C and D). A significantly lower number of macrophages was observed in glomeruli of heparanase-deficient mice 4 days after the induction of anti-GBM glomerulonephritis and 2 days after the induction of LPS glomerulonephritis (Figure 5, C and D). Taken together, heparanase deficiency significantly reduced glomerular leukocyte influx in antiGBM glomerulonephritis and glomerular macrophage influx in both models of experimental glomerulonephritis.

The Presence of Glomerular Platelets Decreases in LPS-Induced Glomerulonephritis and Increases in Anti-GBM Glomerulonephritis

Heparanase during experimental glomerulonephritis may originate from glomerular cells, inflammatory cells, and/or platelets. Therefore, we also evaluated the glomerular presence of platelets using the platelet-specific marker CD41. At day 2 after LPS administration, the number of platelets was similar for WT and heparanase-deficient mice. Interestingly, in the LPS model, the number of platelets decreased compared with untreated controls in both WT and heparanase-deficient mice (Supplemental Figure S3), which may suggest that in the LPS model platelet-derived heparanase is not contributing to pathology. In anti-GBM glomerulonephritis, in both WT and heparanase-deficient mice, there was a significant increase in the number of glomeruli with aggregates of platelets already 2 hours after administration of anti-GBM serum, which further increased at days 1 and 4 (Supplemental Figure S3). The number of glomeruli with platelet aggregates was significantly reduced in the heparanase-deficient mice 4 days after induction of anti-GBM glomerulonephritis (Supplemental Figure S3).

Heparanase Deficiency Leads to a Reduced Expression of Th1 and Th2 Cytokines in Anti-GBM and LPS Glomerulonephritis

Because we observed a reduced glomerular influx of leukocytes and macrophages in heparanase-deficient mice after induction of experimental glomerulonephritis in both the anti-GBM and LPS model, we analyzed the expression of types 1 and 2 helper T-cell (Th1) and (Th2; promoting) cytokines in the renal cortex of these mice (Tables 2 and 3). The mRNA expression of Th1 (promoting) cytokines TNF- $\alpha$, interferon- $\gamma$, IL-12 $\alpha$, and IL-12 $\beta$ was lower in the heparanase-deficient mice compared with the WT mice in both anti-GBM and LPS-induced 

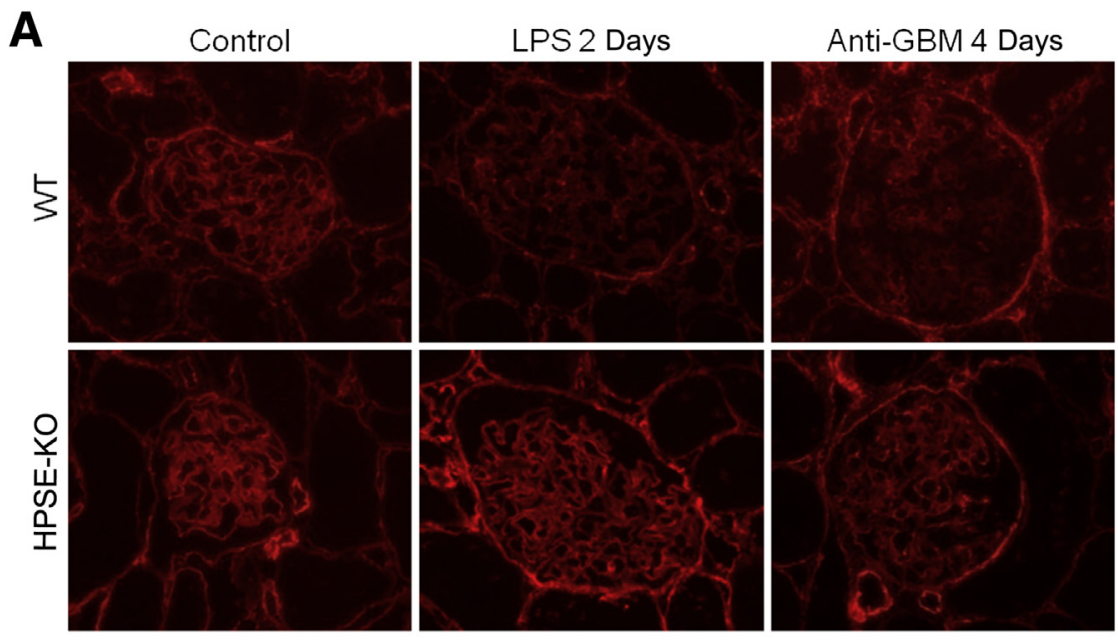

\section{B}
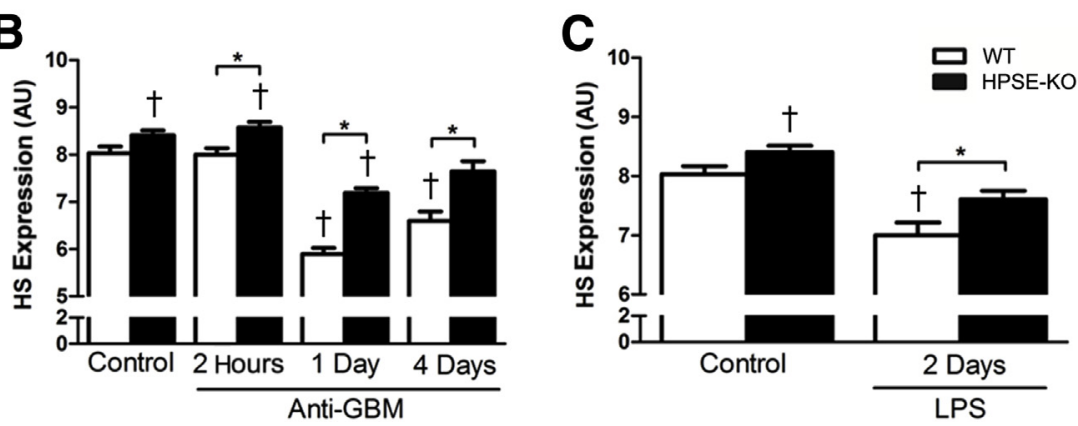

Figure 4 Heparanase deficiency (HPSE-KO) preserves glomerular heparan sulfate (HS) expression in experimental glomerulonephritis. Immunofluorescence staining (A) and semiquantitative analysis ( $\mathbf{B}$ and $\mathbf{C}$ ) of the glomerular expression of the highly sulfated HS domain recognized by the anti-HS antibody HS4C3 show a reduced glomerular expression 1 and 4 days after the induction of anti-glomerular basement membrane (GBM) glomerulonephritis (B) and 2 days after the induction of lipopolysaccharide (LPS) glomerulonephritis in wide-type (WT) mice (C). HS expression is significantly higher in the HPSE-KO mice. D: HS expression inversely correlates with the level of proteinuria $(r=-0.79, P<0.0001)$. ${ }^{*} P<0.05$ versus anti-GBM/LPS-injected WT mice; ${ }^{\dagger} P<0.05$ versus control. Original magnification, $\times 400$ (A). $\mathrm{AU}$, arbitrary unit.

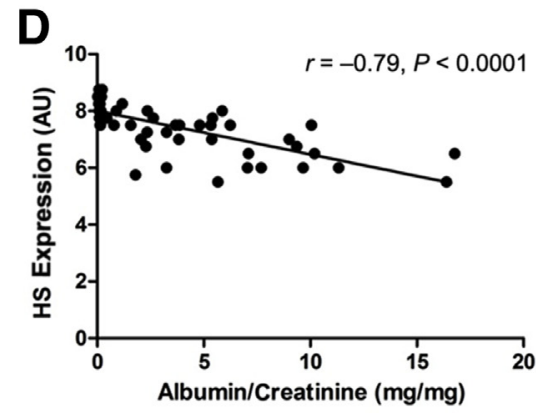

glomerulonephritis. In addition, mRNA expression of the Th2 cytokine IL-10 was also lower in the heparanase-deficient mice in both models. The expression of IL-6, which is both a proinflammatory and anti-inflammatory cytokine, was higher in the heparanase-deficient mice compared with the WT mice in the early phase after induction of anti-GBM glomerulonephritis, but was significantly lower in the heparanase-deficient mice compared with the WT mice after induction of LPS glomerulonephritis. Furthermore, the expression of TNF receptor 1a was lower in the heparanase-deficient mice compared with the WT mice, whereas the expression of TNF receptor $1 \mathrm{~b}$ was comparable to the WT mice. We previously showed that syndecan-1 deficiency aggravates anti-GBM glomerulonephritis, by skewing the Th1/Th2 response. ${ }^{31}$ Consistent with previous results, in the current study, syndecan-1 mRNA expression increased after induction of anti-GBM and LPS glomerulonephritis in the WT mice, whereas syndecan-1 mRNA expression decreased in the heparanase-deficient mice compared with WT mice in the early phase of the anti-GBM model, and was 10-fold lower in the LPS model (Tables 2 and 3). Taken together, heparanase deficiency in experimental glomerulonephritis leads to a less proinflammatory cytokine milieu in the kidney.

\section{TNF- $\alpha$ and LPS Increase Transendothelial Albumin Passage in Vitro}

Next, we evaluated whether TNF- $\alpha$ and LPS regulate heparanase expression in glomerular endothelial cells and podocytes in vitro. Activation of $\mathrm{mGEnC}-1$ with TNF- $\alpha$ or LPS resulted in a twofold and 1.5-fold increased heparanase mRNA expression, respectively (Figure 6A). Activation of mouse podocytes with TNF- $\alpha$ or LPS resulted in a 2.5-fold and twofold increased heparanase mRNA expression, respectively (Figure 6B). At the functional level, activation of mGEnC-1 with TNF- $\alpha$ or LPS resulted in a 1.5- and 1.3-fold increased transendothelial albumin passage, respectively (Figure 6C). Silencing of heparanase expression in mGEnC-1 with shRNA (approximately 60\% knockdown) led to a significant reduction in transendothelial albumin passage after activation with TNF- $\alpha$ or LPS compared with mGEnC-1 transfected with a scrambled shRNA (Figure 6D). These data suggest that heparanase mediates transendothelial albumin passage under inflammatory conditions.

\section{Discussion}

Herein, we showed that heparanase deficiency ameliorated proteinuria, reduced glomerular damage, and reduced the proinflammatory cytokine milieu in the kidney during experimental glomerulonephritis. Induction of experimental glomerulonephritis led to an increased heparanase expression and activity in WT mice, which was associated with a decreased glomerular expression of a highly sulfated HS domain and a significant proteinuria 1 and 4 days after induction of anti-GBM glomerulonephritis and 2 days after induction of LPS glomerulonephritis. This is supported by the in vitro data, where we showed that TNF- $\alpha$ and LPS 

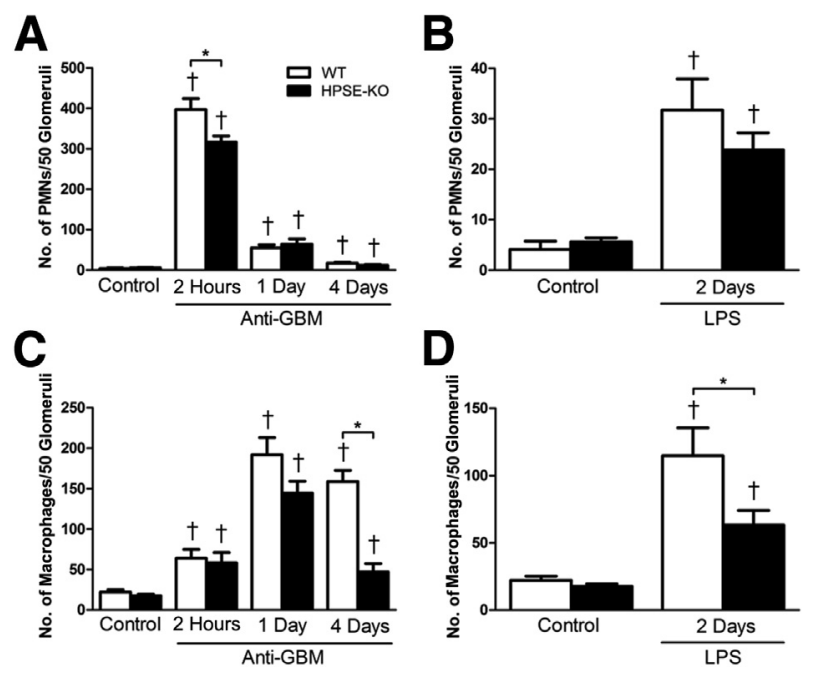

Figure 5 Heparanase deficiency (HPSE-K0) reduces glomerular leukocyte and macrophage influx during experimental glomerulonephritis. A: Glomerular polymorphonuclear granulocytes (PMN) influx, analyzed by immunofluorescence staining, peaks 2 hours after induction of antiglomerular basement membrane (GBM) glomerulonephritis in both wildtype (WT) and HPSE-KO mice, but is significantly lower in the HPSE-KO mice. B: Glomerular PMN influx increases by induction of lipopolysaccharide (LPS) glomerulonephritis, but is not significantly different between WT and HPSE-KO mice. Glomerular macrophage influx significantly increases after induction of both anti-GBM glomerulonephritis (C) and LPS glomerulonephritis (D). HPSE-KO mice show a significantly reduced glomerular macrophage influx 4 days after induction of anti-GBM glomerulonephritis and 2 days after induction of LPS glomerulonephritis. ${ }^{*} P<0.05$ versus anti-GBM/LPS-injected WT mice; ${ }^{\dagger} P<0.05$ versus control.

directly induced heparanase expression in mouse glomerular endothelial cells and mouse podocytes. Moreover, TNF- $\alpha$ and LPS also increased transendothelial albumin passage in vitro, most likely through heparanase-mediated loss of HS expression on glomerular endothelial cells, because silencing of heparanase led to a reduced transendothelial albumin passage. Proteinuria was reduced in the heparanase-deficient mice, which was accompanied by a preserved HS expression in the capillary filter, as measured by an anti-HS antibody recognizing a highly sulfated HS domain. On the other hand, the expression of previously identified inflammatory HS domains that mediate binding of leukocytes and chemokines was reduced in heparanase-deficient mice during disease. ${ }^{13,31,36}$ Our results are consistent with previous studies, where treatment of rats with an anti-heparanase antibody or the heparanase inhibitor PI- 88 reduced proteinuria in passive Heymann nephritis and accelerated anti-GBM disease. ${ }^{25-27}$ In addition, Lygizos et $\mathrm{al}^{29}$ showed that treatment with a heparanase inhibitor attenuated septic acute kidney injury.

We previously showed that heparanase deficiency prevented the development of proteinuria and renal damage in experimental diabetic nephropathy. ${ }^{23}$ However, in contrast to diabetic nephropathy, heparanase deficiency did not completely prevent proteinuria during experimental glomerulonephritis in two different models. Although heparanase deficiency prevented the influx of macrophages during diabetic nephropathy, ${ }^{23,24}$ in the current study, neutrophils and macrophages, although reduced, were still present in the glomeruli of heparanase-deficient mice during experimental glomerulonephritis. Because neutrophils and macrophages are important mediators of proteinuria, glomerular damage, and the local cytokine milieu, this may explain the persisting proteinuria in the heparanase-deficient mice during experimental glomerulonephritis, albeit lower compared with the WT mice.

PMN influx peaked 2 hours after the induction of anti-GBM glomerulonephritis. PMNs are one of the key determinants of proteinuria and glomerular injury in the early phase of antiGBM glomerulonephritis. PMN influx was significantly lower in the heparanase-deficient mice, which could result in a reduced release of proteinases, which can destruct the capillary wall. ${ }^{37}$ This reduced PMN influx may lead to a reduced proteinuria and to an improved renal function after 1 and 4 days.

Table 2 Quantitative mRNA Expression of Sdc-1 and Type 1 and 2 Helper T-Cell Cytokines in Renal Cortex of WT and HPSE-KO Mice during Anti-GBM Glomerulonephritis

\begin{tabular}{|c|c|c|c|c|c|c|}
\hline \multirow[b]{2}{*}{ Variable } & \multicolumn{2}{|l|}{2 Hours } & \multicolumn{2}{|l|}{1 Day } & \multicolumn{2}{|l|}{4 Days } \\
\hline & WT & HPSE-KO & WT & HPSE-KO & WT & HPSE-KO \\
\hline TNF- $\alpha$ & $83.84 \pm 14.38^{* * *}$ & $64.77 \pm 7.84^{* * *}$ & $5.14 \pm 1.18^{* *}$ & $3.89 \pm 0.75^{* *}$ & $5.08 \pm 0.98 * * *$ & $1.87 \pm 0.57^{\dagger}$ \\
\hline IL-6 & $5599 \pm 1144^{* *}$ & $21,269 \pm 3328^{* \dagger}$ & $22.23 \pm 4.30 * *$ & $39.80 \pm 8.63$ & $18.49 \pm 5.86$ & $12.67 \pm 3.47$ \\
\hline IL-10 & $38.70 \pm 5.39^{* * *}$ & $22.85 \pm 2.48^{* * * \dagger}$ & $5.70 \pm 3.42$ & $1.95 \pm 0.35$ & $3.41 \pm 0.79 *$ & $2.50 \pm 0.49^{*}$ \\
\hline IL-12 $\alpha$ & $10.65 \pm 2.82^{* *}$ & $3.47 \pm 0.34^{* * * \dagger}$ & $1.77 \pm 0.30^{*}$ & $0.83 \pm 0.08^{\dagger}$ & $2.30 \pm 0.28^{* *}$ & $0.98 \pm 0.24^{\dagger}$ \\
\hline TNFRSF1b & $9.61 \pm 1.78^{* * *}$ & $12.13 \pm 1.52^{* * *}$ & $1.67 \pm 0.45$ & $2.66 \pm 0.34^{* * *}$ & $3.05 \pm 0.49 * *$ & $2.45 \pm 0.34^{* *}$ \\
\hline Sdc-1 & $2.49 \pm 0.47^{* *}$ & $0.76 \pm 0.15^{\dagger}$ & $1.46 \pm 0.47$ & $0.84 \pm 0.16$ & $2.78 \pm 0.84^{*}$ & $1.55 \pm 0.47$ \\
\hline
\end{tabular}

The means \pm SEM of eight mice per group are depicted.

${ }^{*} P<0.05,{ }^{* *} P<0.01$, and ${ }^{* * *} P<0.001$ versus control mice.

${ }^{\dagger} P<0.05$ HPSE-KO versus WT mice. The relative expression compared with untreated WT or HPSE-KO mice is shown, with both untreated WT and untreated HPSE-KO mice set at 1.

GBM, glomerular basement membrane; HPSE-K0, heparanase deficient; IFN- $\gamma$, interferon- $\gamma$; Sdc- 1 , syndecan- 1 ; TNF- $\alpha$, tumor necrosis factor- $\alpha$; TNFRSF, TNF receptor; WT, wild type. 
Table 3 Quantitative mRNA Expression of Sdc-1 and Type 1 and 2 Helper T-Cell Cytokines in Renal Cortex of WT and HPSE-KO Mice during LPS Glomerulonephritis

\begin{tabular}{lcc}
\hline & \multicolumn{2}{l}{ 2 Days } \\
\cline { 2 - 3 } Variable & WT & HPSE-K0 \\
\hline TNF- $\alpha$ & $18.02 \pm 5.69^{* *}$ & $6.05 \pm 1.13^{* * * \dagger}$ \\
IFN- $\gamma$ & $1.64 \pm 0.38$ & $1.02 \pm 0.29$ \\
IL-6 & $1341 \pm 764^{* *}$ & $282 \pm 141^{*}$ \\
IL-10 & $37.92 \pm 15.41^{*}$ & $22.80 \pm 10.45$ \\
IL-12 $\alpha$ & $4.78 \pm 1.66^{*}$ & $0.90 \pm 0.25^{\dagger}$ \\
IL-12 $\beta$ & $1.68 \pm 0.79$ & $1.55 \pm 0.84$ \\
TNFRSF1a & $4.25 \pm 0.63^{* * *}$ & $2.29 \pm 0.33^{* * \dagger}$ \\
TNFRSF1b & $9.77 \pm 3.08^{*}$ & $14.32 \pm 3.96^{* *}$ \\
Sdc-1 & $19.51 \pm 6.02^{* *}$ & $2.10+0.42^{* \dagger}$ \\
\hline
\end{tabular}

The relative expression compared with untreated WT or HPSE-KO mice is shown, with both untreated WT and untreated HPSE-KO mice set at 1 . The means \pm SEM of eight mice per group are depicted.

${ }^{*} P<0.05,{ }^{* *} P<0.01$, and ${ }^{* * *} P<0.001$ versus control mice.

${ }^{\dagger} P<0.05$ HPSE-KO versus WT mice.

HPSE-KO, heparanase deficient; IFN- $\gamma$, interferon- $\gamma$; LPS, lipopolysaccharide; Sdc- 1 , syndecan- 1 ; TNF- $\alpha$, tumor necrosis factor- $\alpha$; TNFRSF, TNF receptor; WT, wild type.

Heparanase may affect the glomerular PMN influx in several ways. A first possibility is that heparanase may affect the availability of adhesion molecules. This was previously described for lung injury during sepsis. ${ }^{19}$ During acute lung injury, activated heparanase cleaves HS from the pulmonary endothelial glycocalyx, thereby exposing endothelial surface adhesion molecules that allow neutrophil adhesion. A second possible mechanism is by shaping the glomerular endothelial expression of specific inflammatory, $\mathrm{N}$-sulfated and 6-Osulfated, HS domains that mediate leukocyte trafficking. We previously showed that the expression of these inflammatory HS domains is increased on activated glomerular endothelial cells and that they enhance leukocyte adhesion in vitro. ${ }^{13}$ In addition, an increased expression of these inflammatory HS domains was observed 2 hours after induction of anti-GBM glomerulonephritis, at the time leukocyte influx peaked. ${ }^{31,36} \mathrm{It}$ can be speculated that heparanase contributes to the shaping of an inflammatory glycocalyx, thereby promoting the presence of inflammatory HS domains mediating PMN binding. Indeed, we observed a decreased expression of the inflammatory HS domains in the heparanase-deficient mice. A third possible mechanism may involve the HS proteoglycan syndecan-1. A recent study in a myeloma cell line showed that heparanase enhanced the expression and shedding of syndecan- $1 .{ }^{38-40} \mathrm{We}$ previously showed that syndecan-1 deficiency aggravates antiGBM glomerulonephritis by increasing leukocyte influx, suggesting that syndecan-1 has anti-inflammatory properties. ${ }^{31}$ Loss of heparanase may lead to less shedding of syndecan-1, which may lead to a reduced inflammatory response, thereby reducing the PMN influx.

No significant differences in PMN influx were observed between WT and heparanase-deficient mice after induction of LPS glomerulonephritis. The mechanisms of proteinuria and inflammation for anti-GBM and LPS-induced glomerulonephritis may be different. Although the development of our antiGBM glomerulonephritis model is PMN dependent, the development of LPS glomerulonephritis is also mediated by a direct effect of LPS on podocytes. Mice deficient of synaptopodin, an actin-associated protein highly expressed by podocytes, show aggravated proteinuria after LPS administration. ${ }^{41}$ LPS induces the expression of cytoplasmic cathepsin $\mathrm{L}^{42}$ Synaptopodin is a known substrate of cathepsin $\mathrm{L}^{43}$ and degradation of synaptopodin by cathepsin $\mathrm{L}$ resulted in the reorganization of the actin cytoskeleton, foot process effacement, and proteinuria. Consistent with these results, we observed an increased cathepsin L mRNA expression and reduced synaptopodin protein expression (data not shown) after induction of LPS glomerulonephritis. Heparanase deficiency reduced cathepsin L mRNA expression and increased synaptopodin expression (data not shown).

In addition to PMNs, macrophages may play a role in establishing the inflammatory milieu and the subsequent destruction of the glomerular capillary wall. We observed an increased glomerular macrophage influx in both models of experimental glomerulonephritis, which was reduced in the heparanase-deficient mice. Macrophages can be activated by cytokines such as interferon- $\gamma$ and TNF- $\alpha$, bacterial LPS, extracellular matrix proteins, and other chemical mediators. We observed an increased renal expression of the proinflammatory cytokine TNF- $\alpha$ in both experimental models for glomerulonephritis, which was reduced in the heparanase-deficient mice as well. The fact that TNF- $\alpha$, like LPS, can activate
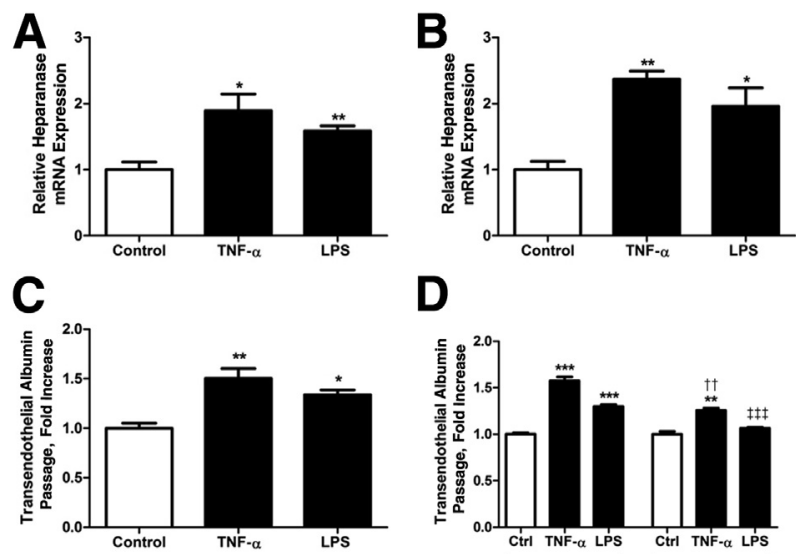

D

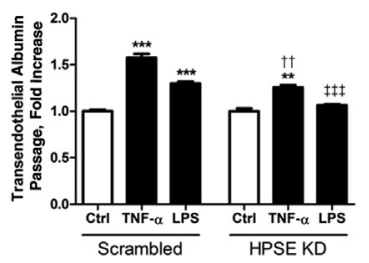

Figure 6 Tumor necrosis factor (TNF)- $\alpha$ and lipopolysaccharide (LPS) induce heparanase expression and increase transendothelial albumin passage in vitro. Activation of mouse glomerular endothelial cells (mGEnC-1; A) and mouse podocytes (B) with TNF- $\alpha$ or LPS for 18 hours results in an increased heparanase mRNA expression. C: Activation of mGEnC-1 with TNF$\alpha$ or LPS for 18 hours increases the passage of albumin across the endothelial monolayer 1.5- and 1.3-fold compared with control, respectively. D: Activation of heparanase-silenced mGEnC-1 with TNF- $\alpha$ or LPS for 18 hours shows a lower transendothelial albumin passage compared with scrambled mGEnC- 1 activated with TNF- $\alpha$ or LPS, respectively. ${ }^{*} P<0.05,{ }^{*} P<0.01$, and ${ }^{* *} P<0.001$ versus control; ${ }^{\dagger \dagger} P<0.01$ versus TNF- $\alpha$ scrambled; ${ }_{\sharp \ddagger \ddagger} P<0.001$ versus LPS scrambled. Ctrl, control; HPSE KD, heparanase silenced. 
macrophages could explain the increased macrophage influx. Recently, Lerner et al ${ }^{22}$ showed that macrophages are more prone to activation by LPS after they have been pretreated with heparanase. In intestinal inflammation, macrophages stimulate epithelial cells, resulting in an increased heparanase expression, which is mainly caused by TNF- $\alpha$. In addition, macrophages can secrete mature cathepsin $\mathrm{L}$, and thereby process and activate heparanase. ${ }^{15}$ In turn, this active heparanase could also induce macrophage activation. Similar results were observed in diabetic nephropathy, where high glucose induced heparanase expression. ${ }^{24}$ Heparanase is processed by cathepsin L produced by tubular cells, and this active heparanase sustains macrophage stimulation, resulting in an increased production of TNF- $\alpha$. Interestingly, in that study, no increase in TNF- $\alpha$ production and macrophage influx was observed in the heparanase-deficient mice. In addition, heparanase expression is normal after induction of LPS glomerulonephritis in TNF receptor 1-deficient mice, which also showed a normal renal function. ${ }^{28}$ Although the exact mechanism of macrophage activation by heparanase is not fully understood, Toll-like receptors (TLRs) may play an important role. ${ }^{44}$ Extracellular HS both inhibits and activates TLR4 signaling and macrophage activation. ${ }^{45-48}$ Activated heparanase can cleave HS on the macrophage cell surface and thereby facilitate ligand binding to TLR $4 .{ }^{22}$ In addition, this cleaved HS can directly stimulate TLR $4,{ }^{45,46,49,50}$ thereby sustaining macrophage activation and promoting renal damage.

In this study, we showed an increased heparanase expression by induction of experimental glomerulonephritis in vivo and stimulation of mGEnC-1 or mouse podocytes with TNF- $\alpha$ or LPS in vitro. Previously, we showed that the early growth response 1 transcription factor can stimulate heparanase expression in kidney cells. ${ }^{23}$ Both TNF- $\alpha$ and LPS can induce the expression of early growth response $1,{ }^{22,51}$ suggesting that heparanase expression is induced via an early growth response 1-dependent mechanism.

Our data are not conclusive with respect to the origin of heparanase in our in vivo models for experimental glomerulonephritis. We can conclude that in the LPS-induced model for glomerulonephritis, heparanase is not derived from platelets, but heparanase may originate from the glomeruli and/or inflammatory cells, such as PMNs and macrophages. In the anti-GBM-induced model, heparanase may be derived from platelets as well. Notably, the anti-GBM model is specifically targeting the glomerulus because of the use of anti-GBM serum, which specifically attracts inflammatory cells and platelets to the glomeruli. Therefore, it may be suggested that heparanase in anti-GBM glomerulonephritis is locally produced by attracted inflammatory cells and platelets, in addition to local production by glomerular cells like podocytes and glomerular endothelial cells. Nevertheless, the exact contribution of cells and tissue in local heparanase production should be addressed in future research by repeating the LPS-induced and anti-GBM-induced experiments in hematopoietic chimera (ie, heparanasedeficient bone marrow in WT mice and vice versa). In addition, although challenging, experiments could be repeated in WT mice with kidney transplants of heparanasedeficient mice and vice versa.

In conclusion, heparanase deficiency significantly ameliorates proteinuria and renal damage during experimental glomerulonephritis by preserving glomerular HS expression, reducing the glomerular influx of leukocytes and macrophages, and affecting the local cytokine milieu. Further studies are required to understand the underlying mechanisms, but overall, heparanase represents an attractive therapeutic target for the treatment of glomerulonephritis.

\section{Supplemental Data}

Supplemental material for this article can be found at http://dx.doi.org/10.1016/j.ajpath.2015.12.008.

\section{References}

1. Tryggvason K, Pettersson E: Causes and consequences of proteinuria: the kidney filtration barrier and progressive renal failure. J Intern Med 2003, 254:216-224

2. Kanwar YS, Farquhar MG: Presence of heparan sulfate in the glomerular basement membrane. Proc Natl Acad Sci U S A 1979, 76: 1303-1307

3. Garsen M, Rops AL, Rabelink TJ, Berden JH, van der Vlag J: The role of heparanase and the endothelial glycocalyx in the development of proteinuria. Nephrol Dial Transplant 2014, 29:49-55

4. Kanwar YS, Farquhar MG: Isolation of glycosaminoglycans (heparan sulfate) from glomerular basement membranes. Proc Natl Acad Sci U S A 1979, 76:4493-4497

5. Groggel GC, Stevenson J, Hovingh P, Linker A, Border WA: Changes in heparan sulfate correlate with increased glomerular permeability. Kidney Int 1988, 33:517-523

6. Kanwar YS, Linker A, Farquhar MG: Increased permeability of the glomerular basement membrane to ferritin after removal of glycosaminoglycans (heparan sulfate) by enzyme digestion. J Cell Biol 1980, 86:688-693

7. van den Hoven MJ, Wijnhoven TJ, Li JP, Zcharia E, Dijkman HB Wismans RG, Rops AL, Lensen JF, van den Heuvel LP, van Kuppevelt TH, Vlodavsky I, Berden JH, van der Vlag J: Reduction of anionic sites in the glomerular basement membrane by heparanase does not lead to proteinuria. Kidney Int 2008, 73:278-287

8. Harvey SJ, Jarad G, Cunningham J, Rops AL, van der Vlag J, Berden JH, Moeller MJ, Holzman LB, Burgess RW, Miner JH: Disruption of glomerular basement membrane charge through podocyte-specific mutation of agrin does not alter glomerular permselectivity. Am J Pathol 2007, 171:139-152

9. Chen S, Wassenhove-McCarthy DJ, Yamaguchi Y, Holzman LB, van Kuppevelt TH, Jenniskens GJ, Wijnhoven TJ, Woods AC, McCarthy KJ: Loss of heparan sulfate glycosaminoglycan assembly in podocytes does not lead to proteinuria. Kidney Int 2008, 74: 289-299

10. van den Born J, van den Heuvel LP, Bakker MA, Veerkamp JH, Assmann KJ, Weening JJ, Berden JH: Distribution of GBM heparan sulfate proteoglycan core protein and side chains in human glomerular diseases. Kidney Int 1993, 43:454-463

11. Rops AL, van der Vlag J, Lensen JF, Wijnhoven TJ, van den Heuvel LP, van Kuppevelt TH, Berden JH: Heparan sulfate 
proteoglycans in glomerular inflammation. Kidney Int 2004, 65 768-785

12. Sarrazin S, Lamanna WC, Esko JD: Heparan sulfate proteoglycans. Cold Spring Harb Perspect Biol 2011, 3. pii: a004952

13. Rops AL, van den Hoven MJ, Baselmans MM, Lensen JF, Wijnhoven TJ, van den Heuvel LP, van Kuppevelt TH, Berden JH, van der Vlag J: Heparan sulfate domains on cultured activated glomerular endothelial cells mediate leukocyte trafficking. Kidney Int 2008, 73:52-62

14. van den Hoven MJ, Rops AL, Vlodavsky I, Levidiotis V, Berden JH, van der Vlag J: Heparanase in glomerular diseases. Kidney Int 2007, $72: 543-548$

15. Abboud-Jarrous G, Atzmon R, Peretz T, Palermo C, Gadea BB, Joyce JA, Vlodavsky I: Cathepsin L is responsible for processing and activation of proheparanase through multiple cleavages of a linker segment. J Biol Chem 2008, 283:18167-18176

16. Ilan N, Elkin M, Vlodavsky I: Regulation, function and clinical significance of heparanase in cancer metastasis and angiogenesis. Int J Biochem Cell Biol 2006, 38:2018-2039

17. Yang Y, Macleod V, Bendre M, Huang Y, Theus AM, Miao HQ, Kussie P, Yaccoby S, Epstein J, Suva LJ, Kelly T, Sanderson RD: Heparanase promotes the spontaneous metastasis of myeloma cells to bone. Blood 2005, 105:1303-1309

18. Parish CR, Freeman C, Hulett MD: Heparanase: a key enzyme involved in cell invasion. Biochim Biophys Acta 2001, 1471: M99-M108

19. Schmidt EP, Yang Y, Janssen WJ, Gandjeva A, Perez MJ, Barthel L, Zemans RL, Bowman JC, Koyanagi DE, Yunt ZX, Smith LP, Cheng SS, Overdier KH, Thompson KR, Geraci MW, Douglas IS, Pearse DB, Tuder RM: The pulmonary endothelial glycocalyx regulates neutrophil adhesion and lung injury during experimental sepsis. Nat Med 2012, 18:1217-1223

20. Li RW, Freeman C, Yu D, Hindmarsh EJ, Tymms KE, Parish CR, Smith PN: Dramatic regulation of heparanase activity and angiogenesis gene expression in synovium from patients with rheumatoid arthritis. Arthritis Rheum 2008, 58:1590-1600

21. Brenchley PE: Antagonising angiogenesis in rheumatoid arthritis Ann Rheum Dis 2001, 60(Suppl 3):iii71-iii74

22. Lerner I, Hermano E, Zcharia E, Rodkin D, Bulvik R, Doviner V, Rubinstein AM, Ishai-Michaeli R, Atzmon R, Sherman Y, Meirovitz A, Peretz T, Vlodavsky I, Elkin M: Heparanase powers a chronic inflammatory circuit that promotes colitis-associated tumorigenesis in mice. J Clin Invest 2011, 121: $1709-1721$

23. Gil N, Goldberg R, Neuman T, Garsen M, Zcharia E, Rubinstein AM, van Kuppevelt T, Meirovitz A, Pisano C, Li JP, van der Vlag J, Vlodavsky I, Elkin M: Heparanase is essential for the development of diabetic nephropathy in mice. Diabetes 2012, 61:208-216

24. Goldberg R, Rubinstein AM, Gil N, Hermano E, Li JP, van der Vlag J, Atzmon R, Meirovitz A, Elkin M: Role of heparanase-driven inflammatory cascade in pathogenesis of diabetic nephropathy. Diabetes 2014, 63:4302-4313

25. Levidiotis V, Freeman C, Tikellis C, Cooper ME, Power DA: Heparanase is involved in the pathogenesis of proteinuria as a result of glomerulonephritis. J Am Soc Nephrol 2004, 15:68-78

26. Levidiotis V, Freeman C, Punler M, Martinello P, Creese B, Ferro V, van der Vlag J, Berden JH, Parish CR, Power DA: A synthetic heparanase inhibitor reduces proteinuria in passive Heymann nephritis. J Am Soc Nephrol 2004, 15:2882-2892

27. Levidiotis V, Freeman C, Tikellis C, Cooper ME, Power DA: Heparanase inhibition reduces proteinuria in a model of accelerated antiglomerular basement membrane antibody disease. Nephrology (Carlton) 2005, 10:167-173

28. Xu C, Chang A, Hack BK, Eadon MT, Alper SL, Cunningham PN TNF-mediated damage to glomerular endothelium is an important determinant of acute kidney injury in sepsis. Kidney Int 2014, 85: $72-81$
29. Lygizos MI, Yang Y, Altmann CJ, Okamura K, Hernando AA, Perez MJ, Smith LP, Koyanagi DE, Gandjeva A, Bhargava R, Tuder RM, Faubel S, Schmidt EP: Heparanase mediates renal dysfunction during early sepsis in mice. Physiol Rep 2013, 1:e00153

30. Zcharia E, Jia J, Zhang X, Baraz L, Lindahl U, Peretz T, Vlodavsky I, Li JP: Newly generated heparanase knock-out mice unravel coregulation of heparanase and matrix metalloproteinases. PLoS One 2009, 4:e5181

31. Rops AL, Gotte M, Baselmans MH, van den Hoven MJ, Steenbergen EJ, Lensen JF, Wijnhoven TJ, Cevikbas F, van den Heuvel LP, van Kuppevelt TH, Berden JH, van der Vlag J: Syndecan1 deficiency aggravates anti-glomerular basement membrane nephritis. Kidney Int 2007, 72:1204-1215

32. van de Westerlo EM, Smetsers TF, Dennissen MA, Linhardt RJ, Veerkamp JH, van Muijen GN, van Kuppevelt TH: Human single chain antibodies against heparin: selection, characterization, and effect on coagulation. Blood 2002, 99:2427-2433

33. Raats CJ, Bakker MA, Hoch W, Tamboer WP, Groffen AJ, van den Heuvel LP, Berden JH, van den Born J: Differential expression of agrin in renal basement membranes as revealed by domain-specific antibodies. J Biol Chem 1998, 273:17832-17838

34. Rops AL, van der Vlag J, Jacobs CW, Dijkman HB, Lensen JF, Wijnhoven TJ, van den Heuvel LP, van Kuppevelt TH, Berden JH: Isolation and characterization of conditionally immortalized mouse glomerular endothelial cell lines. Kidney Int 2004, 66:2193-2201

35. Mundel P, Reiser J, Zuniga Mejia Borja A, Pavenstadt H, Davidson GR, Kriz W, Zeller R: Rearrangements of the cytoskeleton and cell contacts induce process formation during differentiation of conditionally immortalized mouse podocyte cell lines. Exp Cell Res 1997, 236:248-258

36. Rops AL, Loeven MA, van Gemst JJ, Eversen I, Van Wijk XM, Dijkman HB, van Kuppevelt TH, Berden JH, Rabelink TJ, Esko JD, van der Vlag J: Modulation of heparan sulfate in the glomerular endothelial glycocalyx decreases leukocyte influx during experimental glomerulonephritis. Kidney Int 2014, 86:932-942

37. Assmann KJ, Tangelder MM, Lange WP, Schrijver G, Koene RA: Anti-GBM nephritis in the mouse: severe proteinuria in the heterologous phase. Virchows Arch A Pathol Anat Histopathol 1985, 406: 285-299

38. Ramani VC, Purushothaman A, Stewart MD, Thompson CA, Vlodavsky I, Au JL, Sanderson RD: The heparanase/syndecan-1 axis in cancer: mechanisms and therapies. FEBS J 2013, 280:2294-2306

39. Purushothaman A, Uyama T, Kobayashi F, Yamada S, Sugahara K, Rapraeger AC, Sanderson RD: Heparanase-enhanced shedding of syndecan-1 by myeloma cells promotes endothelial invasion and angiogenesis. Blood 2010, 115:2449-2457

40. Yang Y, Macleod V, Miao HQ, Theus A, Zhan F, Shaughnessy JD Jr, Sawyer J, Li JP, Zcharia E, Vlodavsky I, Sanderson RD: Heparanase enhances syndecan-1 shedding: a novel mechanism for stimulation of tumor growth and metastasis. J Biol Chem 2007, 282:13326-13333

41. Asanuma K, Kim K, Oh J, Giardino L, Chabanis S, Faul C, Reiser J, Mundel P: Synaptopodin regulates the actin-bundling activity of alpha-actinin in an isoform-specific manner. J Clin Invest 2005, 115: $1188-1198$

42. Sever S, Altintas MM, Nankoe SR, Moller CC, Ko D, Wei C, Henderson J, del Re EC, Hsing L, Erickson A, Cohen CD, Kretzler M, Kerjaschki D, Rudensky A, Nikolic B, Reiser J: Proteolytic processing of dynamin by cytoplasmic cathepsin $\mathrm{L}$ is a mechanism for proteinuric kidney disease. J Clin Invest 2007, 117 2095-2104

43. Reiser J, Adair B, Reinheckel T: Specialized roles for cysteine cathepsins in health and disease. J Clin Invest 2010, 120:3421-3431

44. Blich M, Golan A, Arvatz G, Sebbag A, Shafat I, Sabo E, CohenKaplan V, Petcherski S, Avniel-Polak S, Eitan A, Hammerman H, Aronson D, Axelman E, Ilan N, Nussbaum G, Vlodavsky I: Macrophage activation by heparanase is mediated by TLR-2 and TLR- 4 and 
associates with plaque progression. Arterioscler Thromb Vasc Biol 2013, 33:e56-e65

45. Brunn GJ, Bungum MK, Johnson GB, Platt JL: Conditional signaling by Toll-like receptor 4. FASEB J 2005, 19:872-874

46. Goodall KJ, Poon IK, Phipps S, Hulett MD: Soluble heparan sulfate fragments generated by heparanase trigger the release of pro-inflammatory cytokines through TLR-4. PLoS One 2014, 9: e109596

47. Ren JD, Fan L, Tian FZ, Fan KH, Yu BT, Jin WH, Tan YH, Cheng L: Involvement of a membrane potassium channel in heparan sulphate-induced activation of macrophages. Immunology 2014, 141:345-352
48. Akbarshahi H, Axelsson JB, Said K, Malmstrom A, Fischer H, Andersson R: TLR4 dependent heparan sulphate-induced pancreatic inflammatory response is IRF3-mediated. J Transl Med 2011, 9:219

49. Yu L, Wang L, Chen S: Endogenous toll-like receptor ligands and their biological significance. J Cell Mol Med 2010, 14: $2592-2603$

50. Johnson GB, Brunn GJ, Kodaira Y, Platt JL: Receptor-mediated monitoring of tissue well-being via detection of soluble heparan sulfate by Toll-like receptor 4. J Immunol 2002, 168:5233-5239

51. Coleman DL, Bartiss AH, Sukhatme VP, Liu J, Rupprecht HD: Lipopolysaccharide induces Egr-1 mRNA and protein in murine peritoneal macrophages. J Immunol 1992, 149:3045-3051 